\title{
Risks and Benefits of Febuxostat in the Cardiovascular Field, from Trials to the Real World, and State of the Art in Italy
}

\author{
Valerio Massimo Magro ${ }^{1 *}$, Eugenio Boccalone ${ }^{1}$, Michele Caturano $^{2}$, Giovanni Scala ${ }^{3}$ and Walter Verrusio ${ }^{4}$ \\ ${ }^{1}$ Department of Internal Medicine and Geriatrics, University of Campania "Luigi Vanvitelli", Italy \\ ${ }^{2}$ Department of Internal Medicine, AORN San Giovanni di Dio e Ruggi d'Aragona, Salerno, Italy
}

${ }^{3}$ ASL RM2 Home Care Center, Rome, Italy

${ }^{4}$ Department of Cardiovascular, Respiratory, Nephrological, Anesthesiological and Geriatric Sciences, Sapienza University of Rome, Rome, Italy

*Corresponding author: Valerio Massimo Magro, Department of Internal Medicine and Geriatrics, University of Campania “Luigi Vanvitelli”, Italy.
Received Date: August 12, 2019

Published Date: August 27, 2019

\section{Introduction}

Pharmacovigilance is the set of activities whose objective is to provide, on an ongoing basis, the best possible information on the safety of drugs, using the appropriate measures and thus ensuring that the drugs available on the market present, in the authorized use conditions, a favorable risk-benefit ratio for the population. There is a "Pharmacovigilance" section of the body responsible for it (Italian Drug Agency, Agenzia Italiana del Farmaco - AIFA). The doctor can directly send the adverse drug reaction (ADR) report to the pharmacovigilance company manager who inserts in the scheduled time on the national pharmacovigilance network. There is also on the website http://www.agenziafarmaco.gov.it/i a collection of the communications ordered in this part of the site take the part of the "Doctor's Letter", of a letter that is indirectly connected to the prescriber (normally delivered to doctors also by post). When the problem takes on a more general character, concerning an example of a category of drugs, the Doctor's Letter is generally accompanied by other informative material (for example lists of questions and answers) also useful for non-specialist readers. The information may relate to modifications of some prescribed aspects, new knowledge on possible side effects, with regards to the correct use of specific categories of drugs, particular precautions for use, the recommendation to pay attention to possible outbreaks of adverse reactions. Febuxostat is an enzyme inhibitor used to treat chronic hyperuricemia with urate deposition (including history, or the presence, of tophi and / or gouty arthritis) [1,2], approved on the European level of allopurinol and US (European League against Rheumatism, EULAR; National Institute for Clinical Excellence, NICE; American College of Rheumatology, ACR).

\section{Discussion}

Until recently, Italian scientific rheumatologic societies, in consensus among which drafters were also counted, the colleagues belonging to the branch of general medicine [3] aligned themselves with international societies and recommended their use (level of recommendation Ia), exalting the greater efficacy and lower adverse effects compared to allopurinol. However, several known studies showed a higher incidence of cardiovascular (CV) events in cohorts treated with febuxostat compared to those with allopurinol [4], although in a different way between the various studies and without a clear explanation. Another part, hyperuricemia, although it does not currently have a certified role as a cardiovascular risk factor, is raised the threshold and it has been observed that patients suffering from hyperuricemia have a cardiovascular risk found [5]. Up to the years 2016-2017, the data on the cardiovascular safety of this molecule are not aroused for reasons of consumption [6], up to the CARES (Cardiovascular safety of Febuxostat and Allopurinol in patients with gout and cardiovascular morbidity) [7,8] and FAST [9] studies, which dealt with cardiovascular outcomes in patients with cardiovascular disease and disease, bringing out an unfavorable safety profile in CARES (the second study cited is in progress) and sending AIFA an information note addressed to doctors, specified general practitioners. In a recent Sing's et al. study, the authors evaluated the comparative effectiveness of allopurinol and febuxostat for the risk of atrial fibrillation (AF) in the elderly. They used used the 5\% Medicare Beneficiary cohort ( $\geq 65$ years) from 2006 to 2012 to identify people with a new filled prescription for allopurinol or febuxostat without respective medication and 
without AF. They assess whether allopurinol use differed from febuxostat use regarding the hazard ratio of incident AF. They concluded that febuxostat was associated with a higher risk of AF compared with allopurinol in older adults. Increased AF risk was noted with febuxostat $80 \mathrm{mg} /$ day dose and was most evident in the first 6 months of use. Moreover, they said that the AF risk with febuxostat compared with allopurinol was higher in people with pre-existing coronary artery disease (CAD). Allopurinol seemed protective against the risk of $\mathrm{AF}$ and longer duration of allopurinol use was associated with a lower risk of AF than shorter durations of allopurinol use, especially in patients with gout [10].

In the CARES study the authors examined the cardiovascular safety of febuxostat or allopurinol in patients with gout. They conducted a multicenter, double-blind, non inferiority trial involving over 6000 patients with gout at high CV risk. The patients were randomly assigned to receive febuxostat or allopurinol, were stratified according to kidney function and were followed for a median of 32 months. Among patients with gout and cardiovascular disease, treatment with febuxostat resulted in overall rates of major adverse cardiovascular events similar to those associated with allopurinol. Higher all-cause mortality, resulting from an imbalance in cardiovascular deaths, was observed with febuxostat than with allopurinol [11]. In a recent study was investigated the cardiovascular safety of febuxostat and allopurinol in the real world. They conducted a cohort study using the Taiwan National Health Insurance Research Database. They identified new users of febuxostat and allopurinol between April 2012 and December 2015 and they assessed the risk of major adverse CV events (MACEs), venous thromboembolism (VTE), heart failure (HF) hospitalization, AF hospitalization, CV death, and all-cause mortality. In the realworld setting, febuxostat was associated with an increased risk of HF hospitalization, AF hospitalization, and CV death, compared with allopurinol. The elevated risk of HF was found to be robust. Additionally, a dose-response relationship between febuxostat use and adverse CV outcomes was observed [12]. Instead, in a recent study, the physicians evaluated the effects of allopurinol and febuxostat on cardiovascular mortality in elderly patients with mild-to-moderate HF (New York Heart Association - NYHA class I-II-III). The authors made an observational trial in which 255 elderly patients affected by chronic HF were treated with urate lowering drugs on top of optimal medical treatment for HF and followed a median follow-up period of 5.1 years. The starting dose of febuxostat was $80 \mathrm{mg} /$ day, up-titrated to $120 \mathrm{mg} /$ day. The study concluded that febuxostat reduce risk of cardiovascular mortality compared to allopurinol in elderly HF patients with mildto-moderate HF [13].

Other physicians evaluated the impact of initiating allopurinol or febuxostat treatment on major CV events in patients with gout, preexisting CV disease (CVD) or HF, and stage 3 or 4 Chronic Kidney Disease (CKD). Patients with moderate-to-severe CKD and CVD or HF who initiated febuxostat treatment had a significantly lower rate of major CV events than patients who initiated allopurinol [14]. Moreover, in the Xu's et al. study, the authors investigated about delayed treatment effects of xanthine oxidase inhibition on systolic overload-induced left ventricular hypertrophy and dysfunction. They saw that febuxostat attenuated development of left ventricular (LV) hypertrophy and dysfunction in mice when treatment is initiated within 1 hour of transverse aortic constriction (TAC). This study investigated whether a 7-day delay of treatment with febuxostat or allopurinol would reverse TAC-induced changes after onset of HF. Neither treatment significantly affected TAC-induced LV hypertrophy; only febuxostat caused a modest improvement in LV function ( $\sim 10 \%$ increase in LV ejection fraction). However, the purine analog allopurinol tended to increase mortality compared with febuxostat in HF mice [15]. Finally, in the study of Liu et al. was evaluated systematically searched randomized controlled clinical trials in PubMed and EMBASE from January 2005 to March 2018 about adult humans with hyperuricemia that used febuxostat and allopurinol to assess the effects of febuxostat and allopurinol on mortality and adverse reactions in this patient. The meta-analysis consisted of 13 randomized controlled trials with a combined sample size of 13,539 patients. They concluded that febuxostat is associated with a moderately increased risk of cardiac-related mortality in the overall study population, whereas a significant association with increased cardiac-related mortality was not found in the Asian subgroup [16]. Following these observations, in Italy the AIFA has decided to issue an informative note that proscribed the user of febuxostat to patients with pre-existing important cardiovascular disease with the hypouricemic treatment. The presence of previous myocardial infarction, stroke and unstable angina also contraindicated this type of treatment [17]. Prohibition that is currently in force, in the absence of new, more exhaustive evidences.

\section{Conclusion}

There are recent scientific evidences that make the safety of febuxostat less certain than in the past, On the other hand, the efficacy of the molecule, even if undermined by events, is not small, especially in patients whose cardiovascular risk is high due to past events, today appears to be grounds for further studies, which also clarify the positive effects in certain subclusters of patients, with for example those suffering from heart failure and / or chronic kidney disease. In addition to new randomized trials, pharmacovigilance programs and retrospective studies are needed, conducted both in the hospital but also on the territory, to verify the incidence and prevalence of adverse events highlighted in real-world trials of febuxostat, in order to identify with greater certainty the patients at risk and thus optimize treatments.

\section{Acknowledgement}

None.

\section{Conflict of Interest}

No conflict of interest.

\section{References}

1. Chohan S, Becker MA, Mac Donald PA, Chefo S, Jackson RL, et al. (2012) Women with gout: efficacy and safety of urate-lowering with febuxostat and allopurinol. Arthritis Care Res (Hoboken) 64(2): 256-261. 
2. Becker MA, MacDonald PA, Hunt BJ, Jackson RL (2013) Diabetes and gout: efficacy and safety of febuxostat and allopurinol. Diabetes Obes Metab 15(11): 1049-1055.

3. Manara M, Bortoluzzi A, Favero M, Prevete I, Scirè CA, et al. (2013) Italian Society of Rheumatology recommendations for the management of gout. Reumatismo 65(1): 5-24.

4. Waller A, Jordan KM (2017) Use of febuxostat in the management of gout in the United Kingdom. Ther Adv Musculoskelet Dis 9(2): 55-64.

5. Pichiri I, Targher G (2012) Iperuricemia e rischio cardiovascolare. Giornale Italiano dell'Aterosclerosi 2: 39-51

6. Borghi C, Perez-Ruiz F (2016) Urate lowering therapies in the treatment of gout: a systematic review and meta-analysis. Eur Rev Med Pharmacol Sci 20(5): 983-992.

7. White WB, Saag KG, Becker MA, Borer JS, Gorelick PB, et al. (2018) Cardiovascular Safety of Febuxostat or Allopurinol in Patients with Gout. N Engl J Med 378(13): 1200-1210.

8. Müller-Wieland D, Nitschmann S (2018) Cardiovascular risk in gout patients: Cardiovascular Safety of Febuxostat or Allopurinol in Participants with Gout and Cardiovascular Comorbidities (CARES) Internist (Berl) 59(11): 1224-1228.

9. Thomas M MacDonald, Ian Ford, George Nuki, Isla S Mackenzie, Raffaele De Caterina, et al. (2014) Protocol of the Febuxostat versus Allopurinol Streamlined Trial (FAST): a large prospective, randomised, open, blinded endpoint study comparing the cardiovascular safety of allopurinol and febuxostat in the management of symptomatic hyperuricaemia. BMJ Open 4(7): e005354.

10. Singh JA, Cleveland JD (2019) Comparative effectiveness of allopurinol and febuxostat for the risk of atrial fibrillation in the elderly: propensity-matched analysis of Medicare claims data. European Heart Journal Pp. 1-10.

11. White WB, Saag KG, Becker MA, Borer JS, Gorelick PB, et al. (2018) Cardiovascular Safety of Febuxostat or Allopurinol in Patients with Gout. N Engl J Med 378: 1200-1210.

12. Su CY, Shen LJ, Hsieh SC, Lin LY, Lin FJ, et al. (2019) Comparing Cardiovascular Safety of Febuxostat and Allopurinol in the Real World: A Population-Based Cohort Study. Mayo Clin Proc 94(7): 1147-1157.

13. Cicero AFG, Cosentino ER, Kuwabara M, Degli Esposti D, Borghi C, et al. (2019) Effects of allopurinol and febuxostat on cardiovascular mortality in elderly heart failure patients. Intern Emerg Med.

14. Foody J, Turpin RS, Tidwell BA, Lawrence D, Schulman KL, et al. (2017) Major Cardiovascular Events in Patients with Gout and Associated Cardiovascular Disease or Heart Failure and Chronic Kidney Disease Initiating a Xanthine Oxidase Inhibitor. Am Health Drug Benefits 10(8): 393-401.

15. Xu X, Zhao L, Hu X, Zhang P, Wessale J, et al. (2010) Delayed treatment effects of xanthine oxidase inhibition on systolic overload-induced left ventricular hypertrophy and dysfunction. Nucleosides, Nucleotides and Nucleic Acids 29: 306-313.

16. Liu CW, Chang WC, Lee CC, Shau WY, Hsu FS, et al. (2019) The net clinical benefits of febuxostat versus allopurinol in patients with gout or asymptomatic hyperuricemia e A systematic review and meta-analysis. Nut Metab Cardiovasc Dis S0939-4753(19): 30245-30245.

17. (2019) National Federation of Medical Orders and Surgeons and Dentists (Federazione Nazionale degli Ordini dei Medici e dei Chirurghi e degli Odontoiatri, FNOMCEO). Communication number 97. Subject: Information note on Adenuric (febuxostat). 